\title{
Agarose Gel Electrophoresis
}

National Cancer Institute

\section{Source}

National Cancer Institute. Agarose Gel Electrophoresis. NCI Thesaurus. Code C16537.

A type of electrophoresis that uses a matrix of highly purified ag ar to separate large

molecules. 